This item was submitted to Loughborough's Research Repository by the author.

Items in Figshare are protected by copyright, with all rights reserved, unless otherwise indicated.

\title{
Design and performance of a flexible metal mountable UHF RFID tag
}

PLEASE CITE THE PUBLISHED VERSION

http://dx.doi.org/10.1109/ECTC.2015.7159895

PUBLISHER

(c) IEEE

VERSION

AM (Accepted Manuscript)

\section{PUBLISHER STATEMENT}

This work is made available according to the conditions of the Creative Commons Attribution-NonCommercialNoDerivatives 4.0 International (CC BY-NC-ND 4.0) licence. Full details of this licence are available at: https://creativecommons.org/licenses/by-nc-nd/4.0/

\section{LICENCE}

CC BY-NC-ND 4.0

\section{REPOSITORY RECORD}

Kaur, Navjot, Diana M. Segura-Velandia, W.G. Whittow, David Barwick, Ehidiamen Iredia, Neil Parker, Neil Porter, Paul P. Conway, and Andrew A. West. 2019. "Design and Performance of a Flexible Metal Mountable UHF RFID Tag". figshare. https://hdl.handle.net/2134/20197. 


\title{
Design and Performance of a Flexible Metal Mountable UHF RFID
}

\author{
Navjot Kaur ${ }^{1}$, Diana Segura Velandia ${ }^{1}$, William Whittow ${ }^{1}$, Dave Barwick ${ }^{2}$, Ehidiamen Iredia ${ }^{2}$, Neil Parker ${ }^{2}$, Neil Porter ${ }^{2}$, \\ Paul P.Conway ${ }^{1}$, Andrew A.West ${ }^{1}$ \\ ${ }^{1}$ Wolfson School of Mechanical \& Manufacturing Engineering, Loughborough University, Loughborough, UK \\ ${ }^{2}$ The Centre for Process Innovation, Sedgefield, UK \\ E-mail: n.kaur@lboro.ac.uk, Phone: +44-1509-227678
}

\begin{abstract}
The large number of requirements and opportunities for automatic identification in manufacturing domains such as automotive and electronics has accelerated the demand for item-level tracking using radio frequency identification technology. End-users are interested in solutions that enable a range of objects to be identified without changing the structure of the objects. Passive UHF RFID systems have drawn considerable attention due to their long read range, high data rates and small antenna sizes. However, it is difficult to tag metallic objects using a passive UHF RFID tag due, for example, to the impact of reflected signals from the conducting material on the tag antenna performance. The overall reliability of an RFID system depends significantly on the tag antenna performance, which is responsible for receiving and transmitting the $\mathrm{RF}$ power to and from the RFID reader. Designing an RFID tag, which can be mounted successfully on metallic objects and meet the end-user requirement is a major research challenge. The aim of the research outlined in this paper is to determine the effects of the harsh manufacturing environments in which RFID tags are used and compare the performance of a range of RFID tag substrates that can be used to isolate the RFID tag antenna from metallic objects. The goal is to determine which substrates allow RFID tags to operate efficiently when attached to metallic, lossy or dielectric objects whilst being low cost, high performance, flexible and in some cases reusable solutions. The main focus is to design a flexible, metal mountable RFID tag by optimising design parameters
\end{abstract} such as complex impedance, bandwidth and size. An investigation and analysis of the effects of metallic surfaces on the performance of the tag antennas is presented in this paper. Parameters that are investigated are the distance between the metal surface and the tag, the thickness of the metallic plate and the type and thickness of the antenna substrate. It has been observed that the return loss of the tag antenna is significantly increased $(\mathrm{D}=-\mathrm{n} \mathrm{dB})$ and the resonance frequency shifted $(\mathrm{Df}=\mathrm{m} \mathrm{Hz}$ ) from the expected operating frequency as the tag antenna is located closer (Dm range from $\mathrm{x}-\mathrm{y} \mathrm{cm}$ ) to the metal. When a substrate with a high dielectric coefficient is sandwiched between the tag and the metallic surface, the orientation of the induced current is influenced by the thickness of the material with the result that the reflected radiation can be strengthened. A flexible RFID tag with a substrate consisting of an elastomer / polymer has been investigated. The tag has been designed, modelled and simulated using the CST electromagnetic field modelling software and the performance is evaluated in terms of the shift in resonance frequency, return loss and bandwidth. Paper, PET and FR-4 substrates integrated with a number of different conductive inks and inkjet/screen printing techniques, have also been investigated as potential optimal solutions for the high volume manufacture of UHF RFID tag suitable for metal objects. The research details the design, engineering and performance of a number of RFID tags when applied to various automotive assets / components such as stillages, crankshafts and fuel injectors used in engine assembly plants, and through the supply chain.

\section{Introduction}

In recent years, passive radio frequency identification (RFID) technology has overcome many of the limitations of the current automatic identification systems such as bar-code technology, biometric identification, machine-vision and active transmitters. RFID technology offers advantages in many applications as it eliminates the need of line-of-sight for its operation, can be read when soiled or dirty and can be used to update/write information on it. For manufacturers this benefits directly into cost reductions as labor intensive tasks can be carried out faster and more accurately. Several frequency ranges are in common use in RFID technology. Low frequency (LF) inductive tags operate at $125-134 \mathrm{KHz}$ or 13.56 MHz, which can easily penetrate through water or metals. However, due to inductive coupling between the tag and the reader antenna, the read range is limited to few centimeters only. UHF (860-960 MHz) and microwave $(2.45$ $\mathrm{GHz}$ ) frequencies have drawn considerable attention in industrial applications, due to their long read range, radiative coupling, high data rates and small antenna sizes compared to LF or HF RFID frequency band. The use of passive UHF RFID technology is increasing the most rapidly, due to the low price, good standardisation situation and sufficient performance (3-4 $\mathrm{m}$ reading range) [5]. However, passive UHF tags have underperformed in metal rich environment limiting their utility [14]. Unlike other conventional identification systems, RFID tags are relatively low-power devices operating on the principle of energy-scavenging. The system works on the basis of electromagnetic interaction between the reader and the tags and the read range (maximum distance between the tag and the reading device) of a low-cost RFID tag can extend to few meters. The overall performance of RFID system depends on the tag antenna performance, which is mainly responsible for the energy scavenging. A tag is usually attached directly to the object to be identified and the tag performance is significantly affected by the electromagnetic properties of the materials in contact or the surrounding metal-rich harsh environment. In particular, when a tag is placed near conducting surface, the tag antenna is detuned from its designed frequency of operation and in worst cases, tags may be unreadable at normal ranges. Problems due to antenna detuning are discussed in [1].

RFID technology can be used in supply chain management and factory automation to track items, containers and forklift 
by attaching tags on them. To withstand extreme harsh conditions in manufacturing industry, a cost-effective tagging solution with necessary physical, chemical and RF properties is required. Technically, RFID tags should be able to operate efficiently when mounted on metallic assets and provide reliable read rates when loaded on a forklift passing through a portal equipped with reader antenna. The information once stored on an RFID tag can be updated locally, providing fast local data maintenance and quality assurance of products. RFID system facilitates interoperability on different hierarchical levels within an enterprise ranging from field level to various higher level applications; for example, information captured through RFID portals can provide the location information of an asset virtually. The data captured using RFID tags can be combined with the planning information and processed into appropriate information to be used at different stages of the process. However, the higher investment costs for RFID technology have slowed down their adaptation in industries. This paper investigates a feasibility study of tagging assets within an automotive manufacturing plant. In-plant asset tracking using RFID technology is a major challenge in terms of feasibility, cost and harsh environment. A novel solution to tag industry standard container/stillage is proposed in this paper.

\section{RFID on Metal: Present State-of-The-Art}

Fig. 1 shows the basic structure of a passive RFID tag. The performance of RFID tag depends on several factors such as the tag position, direction of the tag, angle of reader antenna, material of the object, surrounding environment and speed of the object [2]. If an RFID tag is attached to a metallic object, the antenna performance is significantly degraded because of the reactance variation on the antenna impedance. Therefore, the tags either need to be specifically designed for metal or attached with special spacers or isolators. Various methods have been proposed by different researchers to isolate RFID tags from conducting materials [4], [6], [7]. A patch antenna with an electromagnetic band gap ground plane is proposed in [9]. However, these tags are much more expensive and difficult to commercialize as they require new antenna and infrastructure. The tag antenna problems for RFID systems in close proximity to objects such as metal and dielectrics (water filled container) are presented in [8]. They have reported that there is a decrease in reading distance and changes in the tag antenna impedance when the tag is close to the metal surface. The tag antenna gain is significantly decreased when it is placed near a metal surface. The change in antenna parameters due to variation in separation between tag antenna and metal surface is discussed in [1]. It has been reported that there is not much effect on the antenna performance when placed at a distance more than the quarter wavelength of the antenna's resonance frequency. The simulation results also show that the thickness of the metallic plate does not affect the performance of the tag antenna. Many metal mountable passive UHF RFID tags have been proposed in literature in recent years. Those tags are designed based on electronic band gap (EBG) substrate [9], cavity backed antennas ([3], [13]), high-permittivity Ceramic-Polymer composite substrate [16]. In recent years, many metal mountable passive UHF RFID tags have been designed and made commercially available. However, these tags are much more expensive and require special infrastructure for new antenna design. In this paper, a novel and efficient solution using the lowest cost commercial label tag; capable of performing on metallic surface is proposed.

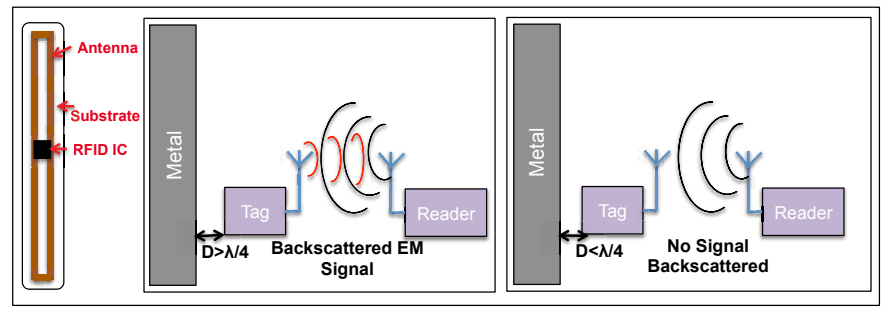

Figure 1. A passive UHF RFID tag and metal interference.

\section{Problem Description and Proposed Method}

The proposed metal mountable tag is designed based on Maxwell's theory of electromagnetic boundary conditions between two different mediums [5]. Boundary between two mediums is shown in Fig. 2.

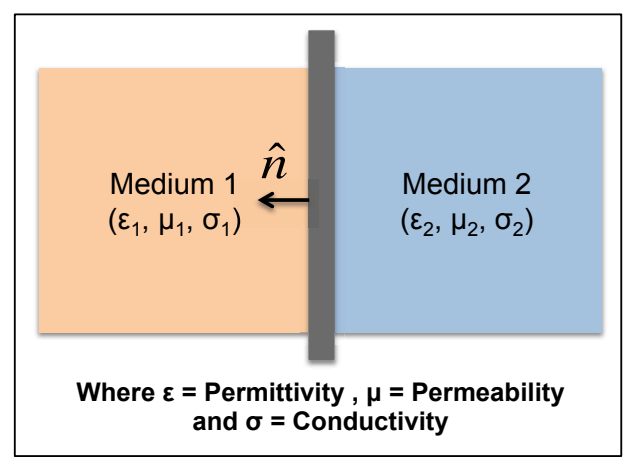

Figure 2. Boundary conditions between two mediums [6].

If medium 1 is considered as a perfect electric conductor metallic medium with infinite conductivity, the boundary conditions for electric (E, D) and magnetic field (B, H) tangential and normal components become []:

$$
\begin{aligned}
& \hat{n} \times E_{1}=0 \\
& \hat{n} \times D_{1}=\rho_{s} \\
& \hat{n} \times H_{1}=J S \\
& \hat{n} \cdot B_{1}=0
\end{aligned}
$$

Where $\rho_{s}=$ surface charge density and $J_{s}=$ surface current density. It signifies that there are only tangential components of the magnetic field and there are no tangential components of the electric field near a perfect conductor. In addition, the magnetic field will be almost doubled near the metallic surface. Therefore, all the electromagnetic components are not available near a perfect metal conductor. Therefore, a passive RFID tag cannot be read at all when attached to a metal object. This research investigates feasibility of attaching a passive UHF RFID tag by introducing isolation/spacer between the tag and the metallic surfaces. A dielectric material introduced between the tag and the metal surface will allow the electromagnetic waves radiate on top of the tag. A novel approach for designing a metal mountable tag is shown in Figure 3. The proposed tag is designed using a commercial paper based label RFID tag (Alien Squiggle ALN-9640), and an elastomer Silicone attached on back of the RFID tag. The 
thickness of Silicone ranges from $10-40 \mathrm{~mm}$ and its relative permittivity is 4 . Use of an elastomer spacer provides a flexible tagging solution. Tag with $40 \mathrm{~mm}$ Silicone is encapsulated in Acrylonitrile Butadiene Styrene (ABS) package for ruggedness and protection in industrial harsh environment. Packaged tag when attached to an industry standard stillage/pallet was readable up to a distance of $8 \mathrm{~m}$ in metal rich environment. The design, cost and performance of proposed RFID tag is compared with commercially available metal mountable tags.

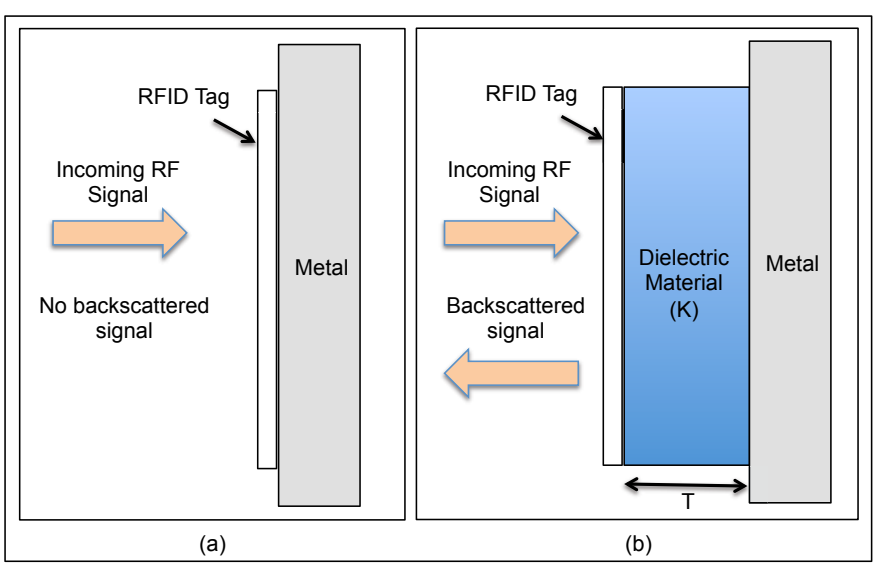

Figure 3. (a) RFID tag on a metal surface (b) proposed method for designing a metal mountable RFID tag.

\section{Novel method for printing passive RFID label tags}

The research in this paper also investigates a printing method for high-volume manufacturing of passive RFID label tag. As shown in Fig., an RFID tag antenna consists of conductors (radiation elements) and dielectric (substrate) [18].The dielectric materials such as paper, plastic or polymers are used as antenna substrate and commonly used low cost materials are polyethylene tetraphthalate (PET) and rigid printed circuit board (PCB) FR-4 (Flame Retardant type - 4). The selection of substrate and conductor ink is a trade-off between cost and performance. Performance of RFID tag antenna based on the antenna trace materials is studied in [15]. It has been reported that Copper $(\mathrm{Cu})$ deposits and Silver (Ag) inks are competitive materials compared to Aluminium for $1 \mathrm{~mm}$ or lesser antenna trace widths [1]. For engineering purposes (simplicity, reliability, cost) it was decided to construct a tag antenna using a flexible polymer PET $(125 \mu \mathrm{m})$. Alien Squiggle tag was initially made of Copper antenna pattern on a paper substrate. Electrical properties of paper and PET are quite similar. However, it has been found that RFID tag performance is significantly improved when printed using Silver ink [17]. Therefore, Squiggle antenna pattern on PET was printed using Silver ink (20 microns). Printing on PET substrate using flexographic printing technique was conducted at CPI, one of our industrial partners involved in this research. RF chip is same for both tags (Higgs3). The performance of the tag was evaluated on an automotive asset used in engine assembly plant and it has been found that the tag performance is almost doubled (results in next section).

\section{Implementation and Experiments}

The approach described above has been implemented and tested using our range-finder software and an Alien
Technology's UHF RFID reader with a circularly polarized antenna (Table I). The distance between the reader antenna and tag was varied and the change in tag performance was recorded in terms of received signal strength indication (RSSI) using range-finder software. For simplicity, maximum read range of different tags was measured when reader antenna and tag antenna were oriented at 0 degree angle. The experiments described here were carried in two different metal rich laboratories based at Loughborough University.

Table I Reader Antenna Details

\begin{tabular}{|c|c|}
\hline Parameter & Description \\
\hline Reader & $\begin{array}{c}\text { Alien ALR9900 Enterprise } \\
\text { Reader }\end{array}$ \\
\hline Reader Antenna Type & Circularly Polarised \\
\hline No. of Antennas & 1 \\
\hline Reader Protocol & Alien Reader Protocol \\
\hline Reading Frequency & 0.5 seconds \\
\hline Antenna Attenuation & $33 \mathrm{dBm}$ \\
\hline Reader Antenna Angle & 0 degrees \\
\hline
\end{tabular}

Performance of RFID tag near a metallic surface

This section presents the testing results showing effect of separation between RFID tag and a metallic plate. As already discussed in previous sections, performance of passive RFID tags is significantly degraded when in contact with a metallic surface; a gap is required between the tag and the metal surface for the tag to respond. The size of the air gap has a large influence on the signal strength and read range of the tag. The wavelength $(\lambda)$ at a particular frequency band can be calculated as :

$$
\lambda=c \text { (speed of light in vacuum) / } f \text { (frequency) }
$$

In this research, commercially available UHF RFID tag Alien Squiggle was selected due to its low cost and easy availability. Wavelength at UHF frequency band in Europe is:

$$
\lambda=346 \mathrm{~mm} \text { at } 866 \mathrm{MHz}
$$
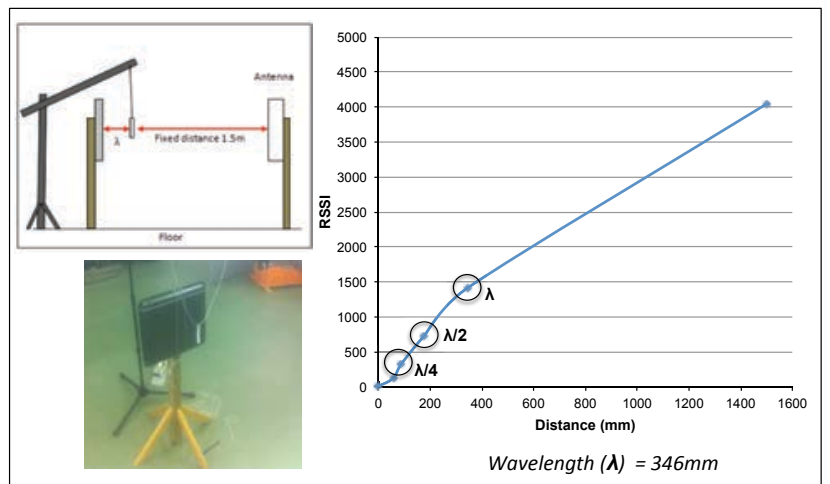

Figure 4. Effect of separation between Squiggle label tag and metallic surface.

Fig. 4 shows the test set up used to analyse the tag performance near a metal plate. The tag was hanged in the air using a string; the reader antenna was fixed $1.5 \mathrm{~m}$ from the tag position while the metal plate $(390 \mathrm{~mm} 3390 \mathrm{~mm} \times 2 \mathrm{~mm})$ was moved in terms of fractions of wavelength $(\lambda, \lambda / 2, \lambda / 4)$ previously calculated. It has been found that when RFID tag was in contact with the metal, there was no read signal due to boundary conditions explained in last sections. As the tag was moved away from the antenna $(\lambda / 6$ and $\lambda / 4)$, the tag 
performance was noticeably improved. The performance of a passive UHF RFID tag is significantly affected if spacing between the tag and metal surface is less than quarter of wavelength $(\lambda / 4)$.

\section{Performance of RFID tag near metal using dielectric spacers/substrates}

This section discusses the effect of inserting dielectric spacers/substrates between RFID tag and meal object. In our testing, two different dielectrics with varying thickness of 10$50 \mathrm{~mm}$ were used: Silicone (relative permittivity $=4$ ), Styrofoam (relative permittivity $=4$ ). The substrates were produced to match the size of the squiggle tag at $10 \mathrm{mmx} 100 \mathrm{~mm}$ surface area with altering thicknesses between $10 \mathrm{~mm}$ up to $50 \mathrm{~mm}$. Tests were conducted with the metal plate in a fixed location and the reader antenna at $1 \mathrm{~m}$ and $2 \mathrm{~m}$ away from the metal surface.

As shown in Fig. 5, the received signal strength values of the tag using Silicone were comparatively higher than the one with Styrofoam. It was noted that $20 \mathrm{~mm}$ Silicone performed quite similar to $40 \mathrm{~mm}$ Styrofoam. This is due to the fact that the relative permittivity of Silicone is higher that Styrofoam. Therefore, in order to use a thinner spacer to achieve a particular tag performance (RSSI), material with higher relative permittivity can be used.

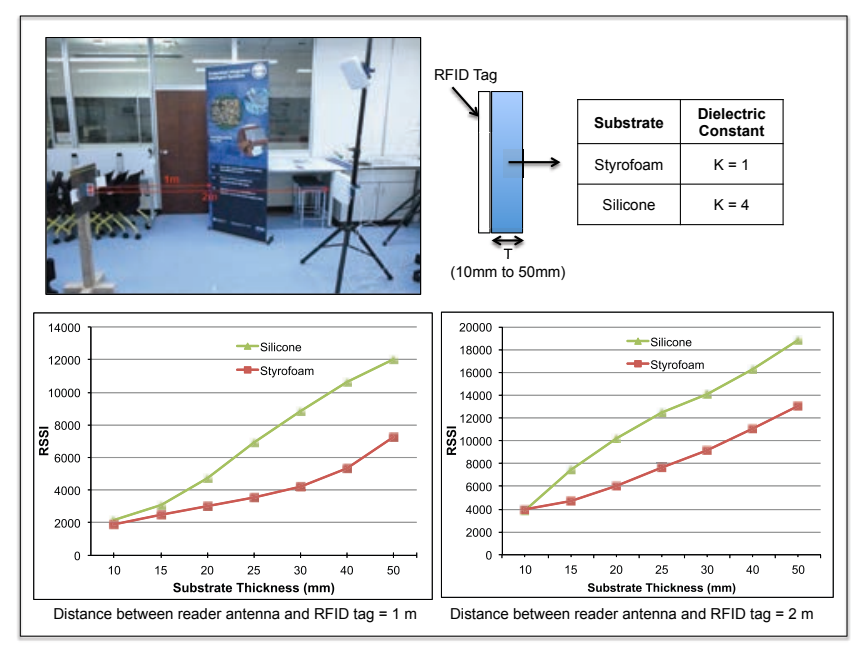

Figure 5. Test set-up and experimental results showing improvement in tag performance with increase in spacer thickness.

\section{Performance of proposed tag on industry standard metal cabinet}

As discussed in the last section, performance of the proposed tag with $40 \mathrm{~mm}$ Silicone was found suitable for tagging metallic objects in RF interfering environment. Therefore, first prototype of the proposed tag was made with $40 \mathrm{~mm}$ Silicone and further tests were conducted on an industry standard metal cabinet in a laboratory-based environment. The tagged cabinet was kept fixed and the reader antenna was moved to a distance of $4.5 \mathrm{~m}$ in intervals of $0.5 \mathrm{~m}$. The maximum read distance was limited due to the size of the lab; however, the RSSI value of proposed tag was more than 3000 at $4.5 \mathrm{~m}$. The tag performance is comparable with a rugged commercial metal mountable tag Omni Dura 3000.

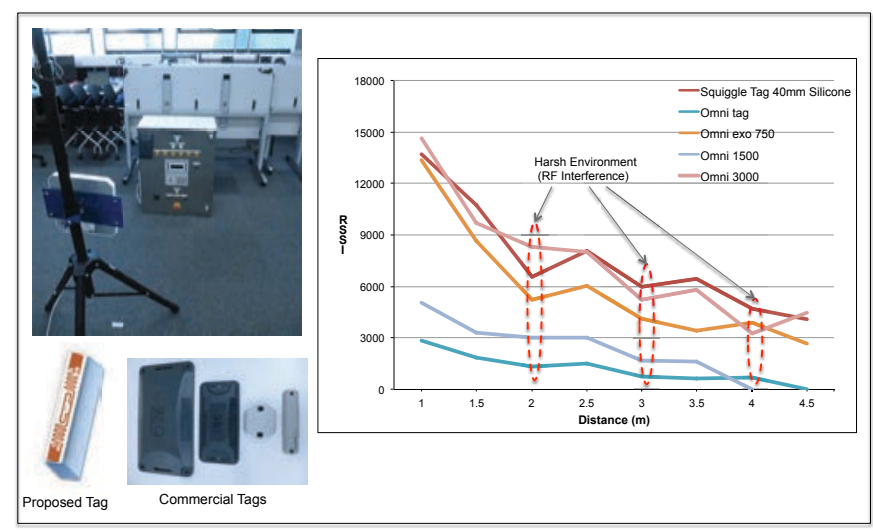

Figure 6. Test set-up and experimental results showing comparison between the proposed tag and commercially available metal mountable tags.

\section{Comparison of prototypes of label tag printed using $\mathrm{Cu}$ and Ag}

The performance of Silver printed PET Squiggle tag was compared with commercial paper Copper printed Squiggle label tag. The tags were attached on standard separator/tray used in engine assembly plant and the reader distance was varied from 1 to $2.5 \mathrm{~m}$. The experimental set-up and the comparison results are shown in Fig. 7.

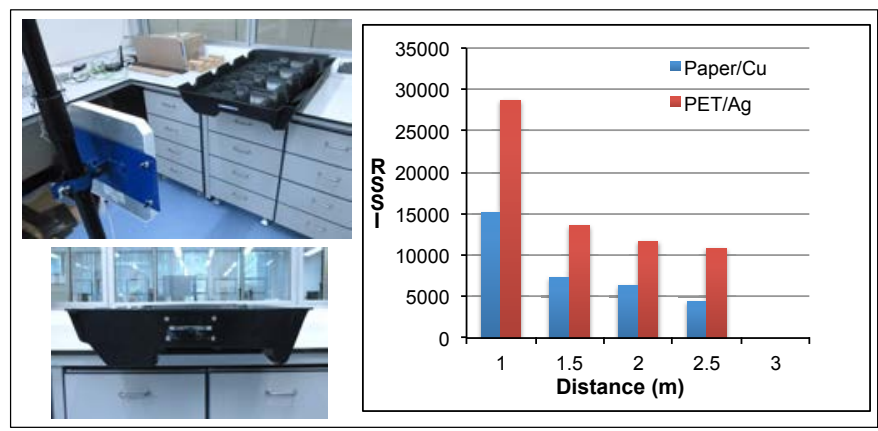

Figure 7. Comparison of Squiggle label tag printed with Paper/Cu and PET/Ag.

It was found that received signal strength of Squiggle label tag was almost doubled when printed using PET/Ag substrate/ink. Two prototypes of metal mountable tags were proposed. As shown in Fig. 9 PET/Ag tag was packaged in ABS and its performance was compared with unpackaged PET/Ag tag keeping a commercial metal mountable tag Omni Dura 3000 as a benchmark. Tests were conducted on a metal plate of size $350 \times 250 \mathrm{~mm}$ with $20 \mathrm{~mm}$ thickness in an anechoic chamber having a walkaway of $4 \mathrm{~m} \times 0.8 \mathrm{~m}$.

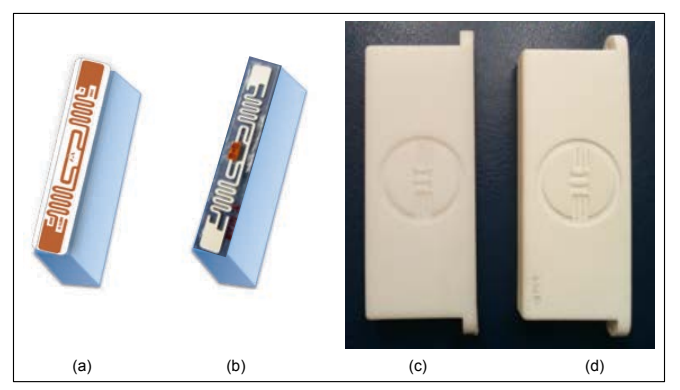

Figure 8. Proposed tag prototypes with 40mm Silicone: (a) Squiggle Paper/Cu label (b) Squiggle PET/Ag label (c) ABS (d) Polyamide packaged. 

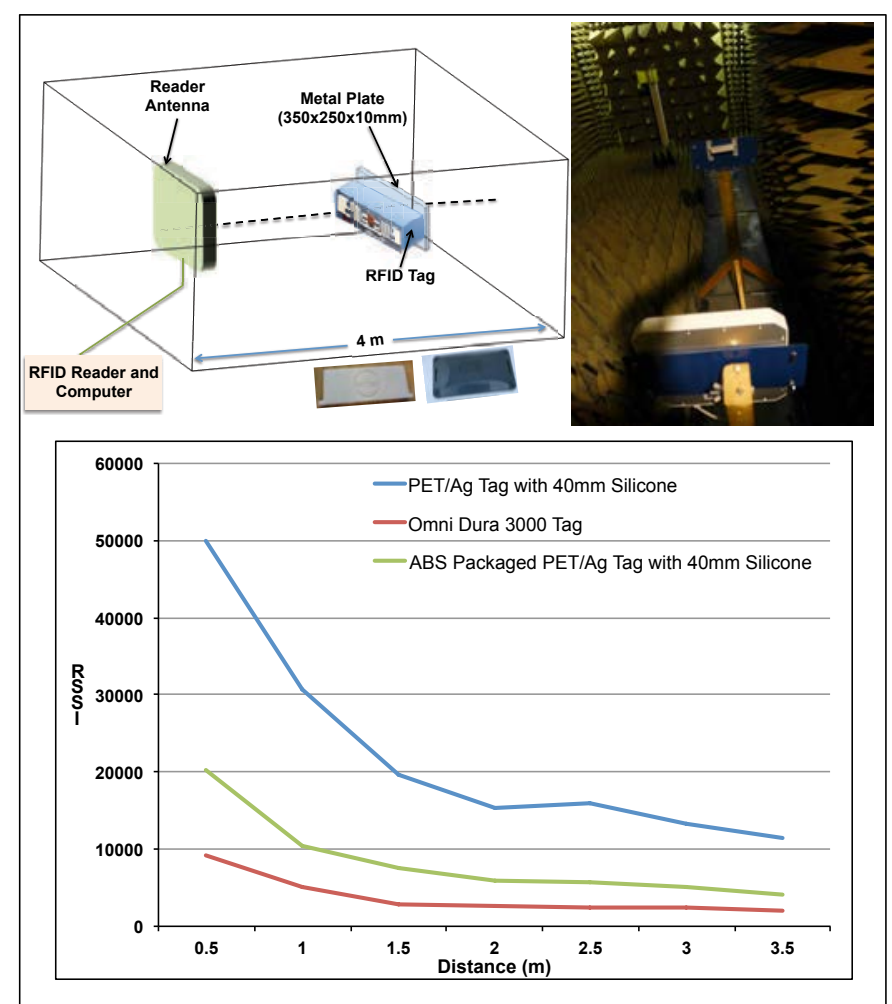

Figure 9. Test set-up and performance of unpackaged and packaged PET/Ag Squiggle label tag.

\section{Case Study: Tagging Industry Standard Stillage/Pallet}

The main aim of this research is to propose a reliable and efficient RF tagging solution for metal containers/stillages used in automotive industry. For this purpose, tag prototype with Squiggle label tag printed with PET/Ag and $40 \mathrm{~mm}$ Silicone was selected. To withstand industrial harsh environment, the tag was encapsulated in rugged package. Fig. 9 shows that packaging material affects the received signal strength, however, the performance of proposed tag is better than Omni Dura 3000. The dimensions and cost comparison of proposed tag and commercial tags is elaborated in Table II. It has been found that the proposed tag offers a compact cost-effective solution for tagging metallic objects. However, to investigate the effect of packaging material, the tag was encapsulated in Polyamide case. The performance of proposed metal mountable tag (PET/Ag $+40 \mathrm{~mm}$ Silicone) packaged in Polyamide case was evaluated when applied to Ford's stillage. The tag was readable up to a distance of $8 \mathrm{~m}$. The experimental set up and results for measurement of maximum read range are shown in Fig. 10. It should be noted that the packaging material affects the signal strength backscattered from the tag. This is due to dielectric properties of ABS and Polyamide which can result in absorption or reflection of RF energy. However, signal penetration through a packaging material should not be affected. Therefore, more packaging materials need to be investigated. The packaged prototype of proposed tag is readable to a distance of more than $8 \mathrm{~m}$, which makes it suitable for tagging Ford's metal stillages enabling their traceability through the supply chain. The tag can be attached using screws, rivets or adhesive tape.

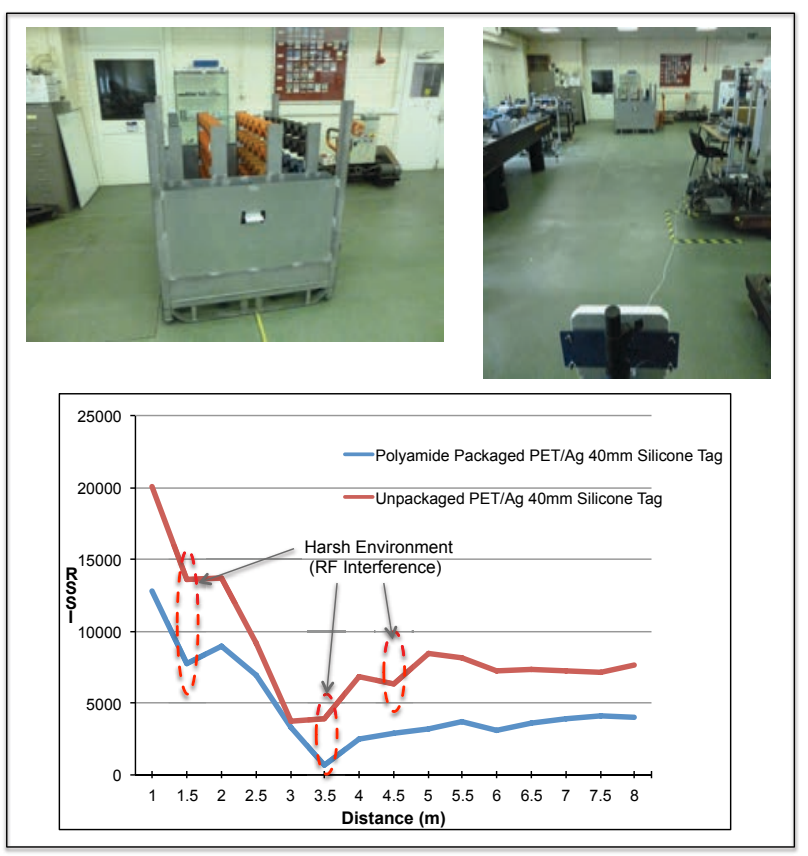

Figure 10. Test set-up and experimental results showing comparison between the proposed tag and commercially available metal mountable tags.

Table II Comparison of Proposed Tag and Commercial Metal Mountable Tags

\begin{tabular}{|c|c|c|c|}
\hline $\begin{array}{c}\text { Tag } \\
\text { Name }\end{array}$ & Structure & $\begin{array}{c}\text { Dimensions (mm) } \\
\text { (LengthxWidthx } \\
\text { Depth) }\end{array}$ & Cost \\
\hline $\begin{array}{c}\text { Omni- } \\
\text { Flexi }\end{array}$ & & 100 X 21 X 7.2 & $£ 0.3 \mathrm{X}$ \\
\hline $\begin{array}{c}\text { Omni Exo } \\
750\end{array}$ & & 51 X 48 X 12.6 & $£ 0.4 \mathrm{X}$ \\
\hline $\begin{array}{c}\text { Omni } \\
\text { Dura 1500 }\end{array}$ & 140 X 66 X 14 & $£ 0.6 \mathrm{X}$ \\
\hline $\begin{array}{c}\text { Omni } \\
\text { Dura 3000 }\end{array}$ & $210 \times 110 \times 21$ & $£ X$ \\
\hline $\begin{array}{c}\text { Proposed } \\
\text { Tag }\end{array}$ & 120 X 15 X 40 & $£ 0.5 \mathrm{X}$ \\
\hline \multicolumn{2}{|c|}{ Cost estimation is for 1000 pieces and X=£8.47 } \\
\hline
\end{tabular}

\section{Conclusions and Future Work}

In this paper, we have proposed a metal mountable RFID tag that offers reliable read range on metallic surfaces. The proposed tag consisted of a commercial label tag (printed with different conductive inks), and a dielectric material/spacer between tag and metallic surface. Silicone material, which has $40 \mathrm{~mm}$ thickness and relative permittivity of 4 , was attached on back side of the RFID tag. The proposed tag is packaged in ABS material for ruggedness and protection. The experimental results using proposed tag are better or comparable to those with more expensive commercial metallic mountable tags. The packaged tag when attached to an industry standard stillage can communicate with readers 
from a distance of $8 \mathrm{~m}$ which makes it suitable for item-level tracking in engine assembly plant or through the supply chain.

The proposed tag design is simple and economical, however, further research needs to be done to reduce the spacer thickness and improve the properties of encapsulation material. For this purpose, either a customised tag antenna pattern or dielectrics with higher relative permittivity need to be investigated. Further experiments replicating the real factory scenario and movement of tagged items through an RFID based portal need to be performed.

\section{Acknowledgments}

The authors wish to express their gratitude to the industrial and academic collaborators of the INTELLICO (Intelligent embedded components for enhanced supply chain observability and traceability) project. The TSB Grant award TP No. 14218-87248 supported this work financially. Also, we thank CPI (Centre for Process Innovation) for their support.

\section{References}

1. T. Dendup, M. S. Hasan, H. Yu, “Analysis of Low Profile UHF RFID Tag Antennas and Performance Evaluation in Presence of a Metallic Surface," In Proc. $5^{\text {th }}$ International Conference on Software, Knowledge Information, Industrial Management and Application (SKIMA), April 8112011 , pp. 1-5.

2. R. Want, "An Introduction to RFID Technology," IEEE Pervasive Computing, vol. 5, pp. 25-33, 2006.

3. K. H. Lee, J. S. Lee, G. Kim, J. Yeo, B. H. Moon, J. Yang, H. C. Kim, "Design of a UHF RFID Metal Tag for Long Reading Range Using a Cavity Structure," In Proceedings of Asia-Pacific Microwave Conference, Macau, China, December, 2008, pp. 1-4.

4. D. D. Deavours, "Improving the Near-Metal Performance of UHF RFID Tags," in Proc. 2010 IEEE International Conference on RFID, Orlando, FL, USA, April 14-16, 2010, pp. 187-194.

5. B. Glover, H. Bhatt, RFID Essentials, 1st ed., O'Reilly Media, CA, USA, 2006, pp. 55-75.

6. C. R. Park, K. H. Eom, "RFID Label Tag Design for Metallic Surface Environments," Sensors 11, no. 1, pp. 938-948, 2011.

7. K. V. S. Rao, S. F. Lam and P. V. Nikitin, "UHF RFID tag for metal containers", Proc. Asia-Pacific Microw. Conf., 2010, pp. $179-182$.

8. D. M. Dobkin and S. M. Weigand, "Environmental effects on RFID tag antennas," In Proc. IEEE MTT-S Int. Microwave Symposium Digest, June 2005, pp. 135 -138.

9. T. H. Loh, "High impedance surface electronic band gap metamaterials: design approach and applications for antenna engineering," In Proc. Automated RF \& Microwave Measurement Society (ARMMS), Nov. 21-22, 2011.

10.J. D. Griffin, G. D. Durgin, A. Haldi and B. Kippelen "RFID tag antenna performance on various materials using radio link budgets," IEEE Antennas Wireless Propagation Letters, vol. 5, no. 1, pp. 247 -250, 2006.

11. M. Stupf, R. Mittra, J. Yeo, and J. R. Mosig, "Some Novel Designs for RFID Antennas and Their Performance
Enhancement with Metamaterials," IEEE Antennas and Propagation Society International Symposium, July 2006, pp. 1023-1026.

12. A. Syed, K. Demarest, D. D. Deavours, "Effects of Antenna Material on the Performance of UHF RFID Tags", in Proc. IEEE International Conference on RFID, March 2007, pp. 57-62.

13. C. M. Seong and D. C. Park, "Design of cavity-backed spiralantennas," Proc. 5th Global Symposium on Millimeter Waves, May 27-30, 2012, pp. $186-190$.

14. L.F. Mo, H. J. Zhang, H. L. Zhou, “Analysis of dipolelike ultra high frequency RFID tags close to metallic surfaces," Journal of Zhejiang University A, vol. 10, no. 8, pp. 1217-1222, 2009.

15. K. V. S. Rao, P. V. Nikitin, and S. F. Lam, "Antenna Design for UHF RFID Tags: A Review and a Practical Application," IEEE Trans. Antennas Propag., vol. 53, no. 12, pp. 3870-3876, Dec. 2005.

16. A. A. Babar, "Inkjet-Printable UHF RFID Tag Antenna on a Flexible Ceramic- Polymer Composite Substrate," IEEE MTT-S Microw. Symp. Dig., pp. 1-3, 2012.

17. P. V. Nikitin, S. Lam and K. V. S Rao, "Low cost silver ink RFID tag antennas", Proc. IEEE Int. Symp. Antennas Propag., vol. 2B, 2005, pp.353 -356.

18. K. Finkenzeller, RFID Handbook, John Wiley \& Sons, New York, NY, USA, 2nd edition, 2003. 